\title{
DESEMPENHO OPERACIONAL DE UM TRATOR AGRÍCOLA EQUIPADO ALTERNADAMENTE COM PNEUS RADIAIS E DIAGONAIS
}

\author{
JACKSON A. BARBOSA ${ }^{1}$, LUCIANO B. VIEIRA ${ }^{2}$, GUTEMBERG P. DIAS ${ }^{2}$, \\ MOACIR DE S. DIAS JÚNIOR ${ }^{3}$
}

\begin{abstract}
RESUMO: A utilização de pneu radial em tratores agrícolas na década de 1950 resultou em redução das perdas de potência oriundas da resistência ao rolamento e patinagem, com conseqüente melhoria no desenvolvimento da tração. Este trabalho teve como objetivo comparar o desempenho operacional de um trator agrícola equipado alternadamente com pneus radiais e diagonais. A avaliação foi realizada a partir de análises de parâmetros indicadores do desempenho dos referidos pneus sob condições específicas de trabalho. $\mathrm{O}$ uso de pneus radiais proporcionou incrementos nos valores de capacidade de tração e potência na barra, diminuição nos valores de consumo específico de combustível e não causou variações no consumo horário de combustível.
\end{abstract}

PALAVRAS-CHAVE: pneus agrícolas, eficiência em tração

\section{OPERATIONAL PERFORMANCE OF AN AGRICULTURAL TRACTOR EQUIPPED ALTERNATELY WITH RADIAL AND DIAGONAL TIRES}

\begin{abstract}
The use of radial tire in agricultural tractors in the decade of 1950 resulted in the reduction of the potency losses originated from the rolling resistance and slippage, with consequent improvement in the traction development. This work had as objective to compare the operational performance of an agricultural tractor, equipped alternately with radial and diagonal tires. The evaluation was accomplished, starting from analyses of indicative parameters of the performance of the referred tires under specific conditions of work. The use of radial tires provided increments in the values of traction capacity and drawbar pull, decrease in values of specific consumption of fuel, and didn't cause variations in the hourly consumption of fuel.
\end{abstract}

KEYWORDS: agricultural tires, traction efficiency.

\section{INTRODUÇÃO}

Além da tradicional ajuda que a roda proporciona ao transporte, é também um elemento que se incorporou pouco a pouco na mecanização agrícola. Os primeiros arados pesados eram dotados de rodas metálicas que auxiliavam no trabalho. Os primeiros tratores agrícolas também utilizavam rodas metálicas, porém dotadas de garras, que serviam como elementos de locomoção. Com a conseqüente diminuição das cargas dinâmicas, o comportamento das rodas teve melhoria mais significativa, convertendo-a em verdadeiro ponto de apoio da mecanização agrícola.

A escolha do pneu adequado para as rodas motrizes de um trator agrícola não deve abranger simplesmente suas três funções principais: suportar carga vertical nos eixos, transmitir potência e produzir força de tração. O problema é mais complexo, pois o trator trabalha em solos que podem

\footnotetext{
${ }^{1}$ Eng ${ }^{\mathrm{o}}$ Agrícola, Prof. Adjunto, Departamento de Agronomia, Faculdades Federais Integradas de Diamantina, FAFEID, Diamantina MG, Fone: (0XX38) 3531.1811, jackson_barbosa@ hotmail.com

${ }^{2}$ Prof. Adjunto, Departamento de Engenharia Agrícola, UFV, Viçosa - MG.

${ }^{3}$ Prof. Adjunto, Departamento de Ciência do Solo, UFLA, Lavras - MG.

Recebido pelo Conselho Editorial em: 26-5-2003

Aprovado pelo Conselho Editorial em: 11-8-2005
} 
apresentar características diferentes. O uso de pneus específicos para determinado tipo de superfície de rolamento permitiria aumentar seu desempenho operacional. No entanto, a variedade dos solos agrícolas é o fator que dificulta o projeto de componentes de tração que funcionem satisfatoriamente em qualquer situação (BARGER et al., 1963).

O índice de $10 \%$ de patinagem situa-se na faixa de obtenção de máxima eficiência trativa em algumas condições de solo agrícola (CORRÊA et al., 1998). O índice de 20\% de patinagem é utilizado por alguns autores (WULFSOHN et al., 1988; DWYER \& FEBO, 1987; GEE-CLOUGH et al., 1977) para expressar o coeficiente líquido de tração quando o objetivo é comparar o desempenho de pneus em campo. O índice de $30 \%$ representa situação de extrema perda de energia pelo rodado, que não é impossível de ser encontrada em operações agrícolas.

A utilização de pneus radiais em tratores agrícolas na década de 1950 resultou em diminuição das perdas de potência oriundas da resistência ao rolamento e patinagem, com conseqüiente melhoria no desenvolvimento da tração. A partir daí, várias pesquisas têm sido desenvolvidas no intuito de estabelecer uma relação entre o dispositivo de tração e o meio no qual ele está operando. Vários estudos também têm sido realizados comparando o desempenho de pneus agrícolas (BARBOSA, 2002; BARBOSA, 1998; DIAS et al., 1993; McMULLAN et al., 1988), contribuindo, dessa forma, para maior entendimento das características operacionais de pneus diagonais e radiais.

O desempenho de pneus radiais para tratores agrícolas, em comparação com diagonais, foi avaliado por CORRÊA et al. (1995a). Os pneus apresentavam dimensões 18.4-34, e as condições de campo se apresentavam como: a) superfície contendo restos de cultura de milho recentemente roçada, densidade global de $1,26 \mathrm{~g} \mathrm{~cm}^{-3}$, teor de água no solo de $12,82 \%$ e índice de cone de $2.476 \mathrm{kPa}$ na camada de 0 a $150 \mathrm{~mm}$, e b) superfície vegetada, densidade global de $1,41 \mathrm{~g} \mathrm{~cm}^{-3}$, teor de água no solo de $15,76 \%$ e índice de cone de $2.740 \mathrm{kPa}$ na camada de 0 a $150 \mathrm{~mm}$. Os parâmetros de desempenho avaliados foram força na barra de tração, potência na barra de tração e coeficiente de tração, sendo todos os parâmetros de desempenho relativos à patinagem dos pneus na faixa de 0 a $30 \%$. Os dados foram analisados pela aplicação do teste $t$ de Student a dados pareados, a 5\% de probabilidade. Os resultados mostraram melhor desempenho do pneu radial para baixos valores de patinagem (valores inferiores a 15\%) para as duas condições de campo. Em valores de patinagem superiores a 15\%, não houve diferença significativa entre os dois tipos de pneus com relação aos parâmetros de desempenho utilizados.

A eficiência trativa de pneus diagonais arrozeiros e pneus radiais no preparo de verão em solos de várzea foi comparada por NEUJAHR et al. (1998), sendo os testes realizados sob condição de solo solto. Os tratamentos foram originados da combinação entre os fatores velocidade de avanço (sendo utilizados dois níveis de velocidade), força de tração e tipo de pneu. As parcelas experimentais foram distribuídas ao acaso, totalizando 36 parcelas de $60 \mathrm{~m}$ de comprimento e $3 \mathrm{~m}$ de largura. Para as condições de realização dos testes, pode-se concluir a ocorrência de menor patinagem e maior eficiência de tração dos pneus radiais comparativamente aos pneus diagonais arrozeiros. Com relação a resistência ao rolamento, constatou-se que a mesma foi menor para os pneus radiais comparado aos pneus diagonais, na maior velocidade testada.

Para avaliar pneus agrícolas radiais e diagonais a campo, com base em análise de tração, NEUJAHR \& SCHLOSSER (2001) utilizaram tratores equipados com instrumentação para obtenção de dados. $\mathrm{O}$ experimento de tração foi desenvolvido em solo de várzea utilizado no cultivo do arroz irrigado por inundação, durante o período em que se realiza o preparo de verão. Foi comparado o desempenho de dois tipos de pneus, radial e diagonal, em condições de superfície de solo firme e sob preparo convencional e em duas velocidades de deslocamento. Os resultados permitiram concluir que os pneus radiais apresentaram menor resistência ao rolamento, principalmente em velocidades maiores, menores índices de patinagem quando comparados aos pneus diagonais submetidos à mesma 
força de tração, menor consumo de combustível quando submetidos a forças de tração maiores que 20 kN, maior coeficiente dinâmico de tração e melhor desempenho em tração dentro dos limites de alta eficiência, ou seja, entre 5 e $20 \%$ de patinagem.

LOPES et al. (2003), com o objetivo de comparar o consumo de combustível de um trator agrícola operando com pneus radial, diagonal e radial de baixa pressão, em duas condições de lastragem e quatro condições de velocidades de deslocamento, montaram experimento em condição de preparo de solo com escarificador. Os resultados evidenciaram vantagens para o trator quando equipado com pneus radiais. Já a condição de lastragem com água nos pneus ofereceu menor consumo específico.

Estima-se que, na produção agrícola, mais da metade do tempo se emprega efetuando atividades de transporte, utilizando como elemento motriz o trator agrícola, também utilizado para arrastar implementos no campo, mediante a transformação da potência do motor em potência de tração da forma mais eficiente possível (LINARES \& JEVENOIS, 1983). Dessa forma, pode-se concluir que qualquer melhoria que puder ser feita com relação ao desempenho dos dispositivos de tração, contribuirá diretamente na eficiência da produção agrícola e para a conservação de energia de origem fóssil.

Este trabalho teve como objetivo comparar o desempenho operacional de um trator agrícola, equipado alternadamente com pneu radial e diagonal.

\section{MATERIAL E MÉTODOS}

Este estudo foi conduzido em um Argissolo Vermelho-Amarelo, localizado no Câmpus da Universidade Federal de Viçosa - MG, com latitude de $20^{\circ} 45^{\prime} 20^{\prime \prime}$ sul, longitude de $42^{\circ} 52^{\prime} 40^{\prime}$ " oeste e altitude média de $650 \mathrm{~m}$.

As análises físicas do solo, realizadas no Laboratório de Geotecnia do Departamento de Engenharia Civil da Universidade Federal de Viçosa, são apresentadas na Tabela 1.

TABELA 1. Características físicas do solo.

\begin{tabular}{lcc}
\hline \multicolumn{1}{c}{ Parâmetro Analisado } & Valor Obtido & Unidade \\
\hline Teor de argila & 0,68 & $\mathrm{~kg} \mathrm{~kg}^{-1}$ \\
Teor de areia & 0,15 & $\mathrm{~kg} \mathrm{~kg}^{-1}$ \\
Teor de silte & 0,17 & $\mathrm{~kg} \mathrm{~kg}^{-1}$ \\
Limite de liquidez & 0,57 & $\mathrm{~kg} \mathrm{~kg}^{-1}$ \\
Limite de plasticidade & 0,42 & $\mathrm{~kg} \mathrm{~kg}^{-1}$ \\
Limite de contração & 0,28 & $\mathrm{~kg} \mathrm{~kg}^{-1}$ \\
Índice de friabilidade & 0,14 & $\mathrm{~kg} \mathrm{~kg}^{-1}$ \\
Densidade do solo & 1,32 & $\mathrm{Mg} \mathrm{m}^{-3}$ \\
Densidade de partículas & 2,73 & $\mathrm{Mg} \mathrm{m}^{-3}$ \\
\hline
\end{tabular}

Média de três repetições

A área onde foi instalado o experimento era usada pelo setor de produção da universidade, estando em pousio por dois anos. Dois meses antes da instalação do experimento, a área foi subsolada, com uso de subsolador com rolo destorroador provido de cinco hastes parabólicas, à profundidade de 0,40 m, sendo eliminada $60 \%$ da cobertura vegetal. Os ensaios ocorreram sob teor de água no solo de $0,42 \mathrm{~kg} \mathrm{~kg}^{-1}$ ( $\left.\pm 5 \%\right)$, cujo valor corresponde a $95 \%$ do teor de água à capacidade de campo.

O delineamento experimental foi inteiramente casualizado, em esquema fatorial $2 \times 3$, com quatro repetições, perfazendo um total de 24 unidades experimentais. Os tratamentos constituíram-se dos 
seguintes fatores: dois tipos de pneus agrícolas - diagonal e radial - e três patinagens dos pneus, sendo 10; 20 e 30\%. Os níveis de patinagem selecionados representam índices possíveis de serem obtidos na maioria das operações agrícolas.

A pressão de inflação utilizada nos pneus foi de $98 \mathrm{kPa}$, segundo orientação de equipe técnica da empresa Pirelli, determinada em função das cargas verticais suportadas pelos pneus, sem comprometimento de sua estrutura.

Cada parcela experimental foi trafegada por um trator agrícola Massey Ferguson 290, 4x2 TDA, de $77 \mathrm{~kW}(105 \mathrm{cv})$ de potência máxima no motor. Peso total de $39,73 \mathrm{kN}$, quando equipado com pneus radiais, e 40,26 kN, quando equipado com pneus diagonais, com distribuição de massa estática de 38 e $62 \%$, respectivamente, para eixos dianteiro e traseiro. $\mathrm{O}$ trator foi operado com pneus diagonais PIRELLI TM 95 18.4-32 no eixo traseiro e PIRELLI TM 95 14.9-24 no eixo dianteiro, e pneus radiais PIRELLI TM 700 510/70 32 no eixo traseiro e PIRELLI TM 200 14.9 R 24 no eixo dianteiro.

Foram impostas cargas à barra de tração pela adoção de um "trator de frenagem" Valmet 110, com o objetivo de obter patinagens dos pneus de 10; 20 e 30\% (ASAE, 1989).

Para cada condição de pneu e patinagem dos rodados motrizes, o trator agrícola deslocou-se por $40 \mathrm{~m}$, em segunda marcha reduzida e $1.750 \mathrm{rpm}$ de rotação no motor, proporcionando velocidade de deslocamento de $4,2 \mathrm{~km} \mathrm{~h}^{-1}$. A rotação e a marcha do trator de frenagem foram alteradas para a obtenção dos diferentes níveis de patinagem.

Durante o tráfego, foram coletados dados referentes à força na barra de tração, patinagem dos pneus dianteiro e traseiro, e consumo de combustível do trator, utilizando-se de sistema de aquisição de dados e respectivos sensores. Juntamente com o volume de combustível, foi monitorada a temperatura, determinando-se, assim, a densidade do diesel em função da temperatura.

A análise estatística constou de análise de variância, em que cada combinação de níveis de fatores foi estimada por um intervalo de confiança, pelo teste de Tukey, a 5\% probabilidade.

\section{RESULTADOS E DISCUSSÃO}

\section{Força de tração}

A força de tração em um trator agrícola é, talvez, o parâmetro mais expressivo para caracterizar a capacidade de tração do veículo (CORRÊA et al., 1995b). Esses mesmos autores citam, ainda, que se encontram na literatura valores de até $33 \%$ a mais de força de tração a favor do pneu radial, dentro da faixa de 0 a $30 \%$ de patinagem.

$\mathrm{Na}$ Tabela 2, apresenta-se a diferença significativa entre o pneu radial e o diagonal ao se comparar a força de tração, com maior valor atribuído ao pneu radial, concordando com CORRÊA et al. (1995a) e NEUJAHR et al. (1998). A força de tração adicional proporcionada pelo pneu radial foi de $15,25 \%$. Ao se analisar o fator patinagem, observa-se diferença significativa entre as mesmas.

\section{Potência na barra}

De maneira semelhante à força de tração, a potência na barra mostrou diferença significativa a favor do pneu radial, o qual apresentou $18,53 \%$ a mais de potência na barra. MYLLER \& TREANOR (1985) encontraram valores correspondentes a 7,6\% a mais de potência na barra para o pneu radial. Com relação à patinagem, não houve diferença significativa entre os níveis de 20 e 30\%, diferindo significativamente do nível de $10 \%$ (Tabela 2). 
TABELA 2. Resultado da análise de variância e do teste de médias dos parâmetros de desempenho do trator agrícola.

\begin{tabular}{|c|c|c|c|c|}
\hline Fatores & $\begin{array}{c}\text { Força de Tração } \\
(\mathrm{kN})\end{array}$ & $\begin{array}{c}\text { Potência na Barra } \\
\qquad(\mathrm{kW})\end{array}$ & $\begin{array}{l}\text { Consumo Horário } \\
\left(\mathrm{L} \mathrm{h}^{-1}\right)\end{array}$ & $\begin{array}{l}\text { Consumo } \\
\text { Específico } \\
\left(\mathrm{g} \mathrm{kW} \mathrm{h}^{-1}\right)\end{array}$ \\
\hline \multicolumn{5}{|l|}{ Pneu (PN) } \\
\hline Radial & $23,13 \mathrm{a}$ & $20,53 \mathrm{a}$ & 13,06 & $544,47 \mathrm{~b}$ \\
\hline Diagonal & $20,07 \mathrm{~b}$ & $17,32 \mathrm{~b}$ & 13,64 & $671,80 \mathrm{a}$ \\
\hline \multicolumn{5}{|l|}{ Patinagem (PT) } \\
\hline $10 \%$ & $15,45 \mathrm{c}$ & $16,40 \mathrm{~b}$ & 11,94 & 641,45 \\
\hline $20 \%$ & $21,69 \mathrm{~b}$ & $19,20 \mathrm{a}$ & 13,51 & 608,12 \\
\hline $30 \%$ & $27,67 \mathrm{a}$ & $21,18 \mathrm{a}$ & 14,60 & 574,84 \\
\hline \multicolumn{5}{|l|}{ Teste F } \\
\hline $\mathrm{PN}$ & $10,67 * *$ & $12,37 * *$ & $0,31 \mathrm{~ns}$ & $9,62 * *$ \\
\hline PT & $56,89 * *$ & $9,29 * *$ & $2,18 \mathrm{~ns}$ & $0,88 \mathrm{~ns}$ \\
\hline PNxPT & $2,80 \mathrm{~ns}$ & $1,97 \mathrm{~ns}$ & $0,28 \mathrm{~ns}$ & $4,09 *$ \\
\hline C.V.\% & 10,60 & 11,79 & 19,13 & 16,53 \\
\hline
\end{tabular}

Em cada coluna, para cada fator, médias seguidas de mesma letra minúscula não diferem pelo teste de Tukey, a $5 \%$ de probabilidade; ns - não significativo ( $p>0,05) ; *$ - significativo $(\mathrm{p}<0,05) ; * *$ - significativo $(\mathrm{p}<0,01)$.

\section{Consumo horário de combustível}

A análise estatística não mostrou diferença significativa para o consumo horário de combustível nas condições experimentais, concordando com LINARES et al. (1992). No presente trabalho, o consumo horário de combustível apresentou valor médio de 13,35 $\mathrm{L} \mathrm{h}^{-1}$ (Tabela 2).

\section{Consumo específico de combustível}

Observa-se, na Tabela 2, que houve diferença significativa entre os pneus, ao se comparar o consumo específico de combustível, com maior valor atribuído ao pneu diagonal, o qual apresentou consumo específico de combustível 23,39\% superior ao pneu radial. LOPES et al. (2003) encontraram menor valor de consumo específico de combustível atribuído ao pneu radial, ao compará-lo ao pneu diagonal e radial de baixa pressão.

Observa-se, ainda, interação significativa entre os fatores pneu e patinagem (Tabela 2). Na Tabela 3, apresenta-se o desdobramento da interação entre esses fatores. Analisando-se o fator pneu dentro de cada patinagem, observa-se que, na patinagem de $10 \%$, ocorreu diferença significativa, com maior valor de consumo atribuído ao pneu diagonal. Já nas condições de patinagem de 20 e 30\%, não houve diferença significativa entre os pneus. Analisando-se o fator patinagem dentro de cada pneu, observa-se que, para o pneu radial, não houve diferença estatisticamente significativa entre as patinagens (Tabela 3). Com relação ao pneu diagonal, observa-se diferença significativa, com maior valor atribuído à patinagem de $10 \%$. A patinagem de $20 \%$ mostrou valor intermediário.

TABELA 3. Desdobramento da interação entre os fatores pneu e patinagem para o consumo específico de combustível $\left(\mathrm{g} \mathrm{kW} \mathrm{h}^{-1}\right)$

\begin{tabular}{lccc}
\hline \multirow{2}{*}{ Pneu } & \multicolumn{3}{c}{ Patinagem } \\
\cline { 2 - 4 } & $10 \%$ & $20 \%$ & $30 \%$ \\
\hline Radial & $510 \mathrm{Ab}$ & $536 \mathrm{Aa}$ & $587 \mathrm{Aa}$ \\
Diagonal & $772 \mathrm{Aa}$ & $680 \mathrm{ABa}$ & $563 \mathrm{Ba}$ \\
\hline
\end{tabular}

Em cada coluna, médias seguidas de mesma letra minúscula e, em cada linha, médias seguidas de mesma letra maiúscula não diferem pelo teste de Tukey, a 5\% de probabilidade. 


\section{CONCLUSÕES}

Ocorreram diferenças significativas a favor do pneu radial, quando comparado ao pneu diagonal, para os parâmetros força de tração e potência na barra.

Não ocorreu diferença significativa com relação ao consumo horário de combustível entre os dois pneus. diagonal.

O pneu radial mostrou menor consumo específico de combustível comparativamente ao pneu

\section{AGRADECIMENTOS}

Às empresas AGCO DO BRASIL e PIRELLI PNEUS, pelo empréstimo do trator agrícola e dos pneus utilizados, respectivamente.

\section{REFERÊNCIAS}

AMERICAN SOCIETY OF AGRICULTURAL ENGINEERS. Uniform terminology for traction of agricultural tractors, self-propelled implements, and other traction and transport devices. St. Joseph: 1989. p.166-8. (ASAE S 296.3)

BARBOSA, J.A. Análise de um modelo para predição de desgaste em pneus motrizes de tratores agrícolas. 1998. 41 f. Dissertação (Mestrado em Mecanização Agrícola) - Universidade Federal de Viçosa, Viçosa - MG, 1998.

BARBOSA, J.A. Efeito do tráfego de pneus agrícolas em propriedades mecânicas de um Argissolo Vermelho-Amarelo. 2002. 119 f. Dissertação (Doutorado em Mecanização Agrícola) - Universidade Federal de Viçosa, Viçosa - MG, 2002.

BARGER, E.L.; LILJEDAHL, J.B.; CARLETON, W.M.; MCKIBBEN, E.G. Tratores e seus motores. São Paulo: Edgar Blucher, 1963. 398 p.

COATES, W. Comparison of bias and radial tractor tires on a soft soil. Transactions of the ASAE, St. Joseph, v.28, n.4, p.1090-3, 1985.

CORRÊA, I.M.; MAZIERO, J.V.G.; MILAN, M. Tração dianteira auxiliar: desempenho em função do pneu dianteiro. In: CONGRESSO BRASILEIRO DE ENGENHARIA AGRÍCOLA, 27., 1998, Poços de Caldas. Anais... Lavras: Sociedade Brasileira de Engenharia Agrícola, 1998. p.154-6.

CORRÊA, I.M.; MILAN, M.; RIPOLLI, T.C.; MAZIERO, J.V.G.; YANAI, K.; MENEZES, J.F. Pneu radial e diagonal: desempenho de tração em um trator com tração dianteira auxiliar. Engenharia Agrícola, Jaboticabal, v.15, p.45-49, 1995 b.

DIAS, G.P.; LIMA, J.S.S.; ESCOBERO, J. F.; DANTAS, A.A.A. Estabilidade lateral de tratores agrícolas. In: CONGRESSO BRASILEIRO DE ENGENHARIA AGRÍCOLA, 22., 1993, Ilhéus. Anais... Ilhéus: Sociedade Brasileira de Engenharia Agrícola, 1993. v.3, p.1838-46.

DWYER, M.J.; FEBO, P. Handbook of agricultural tyre performance. Silsoe: Institut of Engineering Research, 1987. 16 p. (report, 47. AFRC)

GEE-CLOUGH, D.; MCALLISTER, M.; EVERDEN, D.W. Tractive performance of tractor drive tires II. A comparison of radial and cross-ply carcass construction. Journal of Agricultural Engineering Research, Silsoe, v.22, n.4, p.385-95, 1977.

LINARES, P.; JEVENOIS, J. Introducion al sistema terreno-vehiculo. Madrid: Publicación del Departamento de Motores y Máquinas Agrícolas, Universidade Politecnica Madrid, 1983. 85 p. 
LINARES, P.; MARQUEZ, L.; PINZON, R.; VASQUEZ, J. Comparison de neumaticos traseros radiales y convencionales para tractores agrícolas. Rivista di Ingegneria Agraria, Bologna, v.2, p.96$106,1992$.

LOPES, A.; LANÇAS, K.P.; FURLANI, C.E.A.; NAGAOKA, A.K.; CASTRO NETO, P.; GROTTA, D.C.C. Consumo de combustível de um trator em função do tipo de pneu, da lastragem e da velocidade de trabalho. Revista Brasileira de Engenharia Agrícola e Ambiental, Campina Grande, v.7, n.2, p.3826, 2003.

McMULLAN, T.A.G.; PLACKETT, C.W.; PEACHEY, R.O. The behavior of tractor drive tyres at low inflation pressures when reacting high side forces. Journal Agricultural Research, Silsoe, v.39, p.221-29, 1988.

MYLLER, J.P.; TREANOR, R.R. Performance of a four wheel drive tractor equipped with tires. St Joseph: American Society of Agricultural Engineers, 1985. 16 p. (ASAE Paper 85-1048)

NEUJAHR, E.B.; SCHLOSSER, J.F. Comportamento de pneus agrícolas radiais e diagonais em relação à tração [1]. Engenharia Agrícola, Jaboticabal, v.21, n.2, p.180-9, 2001.

NEUJAHR, E.B.; SCHLOSSER, J.F.; DALLMEYER, A.U.; FERREIRA, M.F.; SCHNEIDER, V. Comparação entre pneus diagonais e radiais no preparo de solos de várea. In: CONGRESSO BRASILEIRO DE ENGENHARIA AGRÍCOLA, 27., 1998, Poços de Caldas. Anais... Lavras: Sociedade Brasileira de Engenharia Agrícola, 1998. v.3, p.244-6.

WULFSOHN, D.; UPADHYAIA, S.K.; CHANCELLOR, W.J. Tractive characteristics of radial ply and bias ply tyres in a Califórnia soil. Journal of Therramechanics, Great Britain, v.25, n.2, p.111-34, 1988. 